\title{
IUFOST2006/865 Key issues and open questions in GMO controls
}

\author{
Y. Bertheau ${ }^{\mathrm{a}}$, J. Davison ${ }^{\mathrm{a}}$ and A. Kobilinsky ${ }^{\mathrm{b}}$ \\ ${ }^{a}$ Institut National de la Recherche Agronomique (INRA), Laboratoire de Biologie Cellulaire, Route de Saint \\ Cyr, 78026 Versailles cedex, France \\ ${ }^{\mathrm{b}}$ INRA, MIA, Domaine de Vilvert, 78352 Jouy en Josas, France \\ yves.bertheau@versailles.inra.fr
}

Developments in biotechnology over the last decades have resulted in the release of a range of GMOs (plants but also microorganisms). Many European citizens are expressing a strong reluctance to GMOs and derived products, although a discrepancy between expressed opinions and attitudes of consumption can be observed. The EU implemented a set of regulations, as also done in other countries, for keeping the consumers' freedom of choice, based on clear and reliable mandatory labeling of food- and feedstuffs above the regulatory thresholds of GMOs fortuitous presence.

The current EU legislation established (i) a Community Reference Laboratory (CRL) supported by the European Network of European Laboratories (ENGL), (ii) mandatory traceability of food- and feedstuffs, and (iii) obligation to GMO notifiers to provide the CRL with specific and quantitative detection methods that need to meet specific quality criteria, as well as appropriate reference materials (control samples). This new legislation has taken into account several of the major problems observed by EU research programs.

However, still a number of serious challenges are not covered by the current European legislative framework, e.g. (i) lack of methods for non-EU approved GMOs, (ii) cost-effectiveness of sampling and of (iii) rapid detection methods of GMO, GM taxa and controls (CaMV, Agrobacterium spp., Bacillus spp., etc.) by e.g. PCR multiplexing.

The cost of analyses impacts the cost of end-products and is also a concern for enforcement laboratories whose budgets are generally not extensible while the number of new detection areas (allergens, mycotoxins, etc.) is still growing. Mandatory traceability should thus lead to a reduced need for analytical controls.

Availability of appropriate DSS (Decision Support Systems) that would assist the stakeholders in selecting the most appropriate analytical strategy in a certain environment and at a certain moment could also be a major step forward. The DSS may also be used for interpreting data and taking appropriate decisions in front of particular results, e.g. when suspecting the presence of unknown GMO.

With a few exceptions, most of the issues and open questions discussed here are common to several detection areas and should induce discussions between method developers, analysts and stakeholders. 